\title{
Diet and physical activity in pre-school children: a pilot project for surveillance in three regions of Italy
}

\author{
Claudia Carletti ${ }^{1, *}$, Anna Macaluso ${ }^{1}$, Paola Pani ${ }^{1}$, Margherita Caroli ${ }^{2}$, \\ Mariano Giacchi ${ }^{3}$, Marcella Montico ${ }^{4}$ and Adriano Cattaneo ${ }^{1}$, on behalf \\ of the Project Groupt \\ ${ }^{1}$ Health Services Research and International Health, Institute for Maternal and Child Health, IRCCS Burlo \\ Garofolo, Via dell'Istria 65/1, 34137 Trieste, Italy: ${ }^{2}$ Public Health Nutrition, Local Health Authority BR 1, \\ Francavilla Fontana, Italy: ${ }^{3}$ Department of Public Health, University of Siena, Siena, Italy: ${ }^{4}$ Epidemiology \\ and Biostatistics, Institute for Maternal and Child Health, IRCCS Burlo Garofolo, Trieste, Italy
}

Submitted 22 December 2011: Final revision received 25 May 2012: Accepted 8 June 2012: First published online 16 August 2012

\begin{abstract}
Objective: To test a surveillance system on diet and physical activity based on data gathered at well-child visits.

Design: Cross-sectional data collection on growth, diet, physical activity and sociodemographic variables.

Setting: Offices of 179 paediatricians in three Italian regions.

Subjects: 26898 children for a total of 32915 well-child visits at 1, 3, 5-6, 8-9, 12, 18, 24, 36 and 60-72 months of age.

Results: The BMI Z-score was lower than the WHO standard at 1 and 3 months but higher from 8-9 months onwards. The rates of breast-feeding at 1, 3, 5-6, $8-9$ and 12 months were 88\%, 75\%, 64\%, 52\% and 32\%, respectively, with 5\% and $3 \%$ continuing up to 24 and 36 months. Exclusive breast-feeding was $64 \%$, $54 \%$ and $20 \%$ at 1,3 and $5-6$ months, respectively; $57 \%$ at 5 months and $85 \%$ at 6 months were given complementary foods. Only $8 \%$ and $10 \%$ of children were taking five portions of fruit and vegetables daily, while $47 \%$ and $51 \%$ were consuming sugar-sweetened beverages at 36 and 60-72 months, respectively. At 60-72 months, less than $10 \%$ reported at least $1 \mathrm{~h}$ of moderate-to-vigorous physical activity on $5-7 \mathrm{~d} /$ week, and $32 \%$ watched television or played videogames for more than $2 \mathrm{~h} / \mathrm{d}$, every day. The majority of paediatricians rated the surveillance system as reliable and feasible.

Conclusions: Surveillance for diet and physical activity in pre-school children, with data gathered during well-child visits, is feasible and potentially useful to plan and evaluate activities for the prevention of obesity.
\end{abstract}

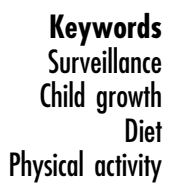

The increasing prevalence of obesity in all age groups is a major public health issue in Europe ${ }^{(1)}$. Childhood obesity is of particular concern because of the health consequences later in life ${ }^{(2)}$. In Italy, an estimated $40 \%$ of adults are overweight or obese, with a north-to-south gradient $^{(3)}$. The same gradient has recently been observed also in school-aged children, with an overall prevalence of $23 \%$ of overweight and $11 \%$ of obesity at 8-9 years of age ${ }^{(4)}$. Data on pre-school children are scarce. Recent reviews have shown that overweight and obesity are prevalent also in early childhood ${ }^{(5)}$, probably due to a number of determinants acting early in life, starting from conception and pregnancy and with an unequal distribution across socio-economic groups ${ }^{(6)}$. With the exception of the data on breast-feeding, very

$\uparrow$ Please see Acknowledgements for a list of members of the Project Group. few data are available on diet and physical activity in pre-school children, one of the main reasons being the difficulty of measuring these variables in this age group. Yet these data are essential for surveillance and to look at changes brought about by interventions aimed at reducing the prevalence of obesity in this age group, which is the focus of many national policies ${ }^{(7,8)}$. The objective of the present study was to test a system for the surveillance of diet and physical activity using data gathered by family paediatricians during well-child visits. The study was part of a larger project for the prevention of overweight and obesity in pre-school children funded by the Centre for the Control of Diseases of the Italian Ministry of Health. The project had three components: (i) the development of an evidence-based kit of good practices for family paediatricians counselling parents on diet and physical activity during well-child visits; 
(ii) the testing of a system for surveillance of a set of indicators on growth, diet and physical activity, including selected sociodemographic variables, to provide family paediatricians and local health authorities information for planning and evaluation purposes; and (iii) the assessment of models for local collaboration with other actors involved in setting the patterns of diet and physical activity in pre-school children. The current paper reports the methods and the results of the system for surveillance of diet and physical activity, i.e. the second component of the project.

\section{Methods}

The project was carried out between May 2007 and September 2010 in three regions: Friuli Venezia Giulia (North), Tuscany (Centre) and Puglia (South of Italy). All family paediatricians in the three regions were informed about the project, with the aim of involving about $10 \%$ of the paediatricians in each region. Participation was on a voluntary basis and eventually entailed thirty-two out of 110 (29\%) paediatricians in Friuli Venezia Giulia, thirtynine out of 440 (9\%) in Tuscany and 108 out of 590 (18\%) in Puglia. In Italy, all children up to 14 years of age are enrolled with a family paediatrician and receive all sorts of care, including optional well-child visits, free of charge. Well-child visits last between 15 and 30 min on average and include a general assessment of health, growth and development, counselling on feeding, advice on prevention (of sudden infant death syndrome, for example), reminders about immunization and screening, information on safety and referral to specialist care as needed. Data are usually gathered for individual use, as opposed to surveillance, and with definitions and methods that are not standardised.

During the project, data on growth, diet, physical activity and sociodemographic variables were collected by means of structured interviews with parents at the nine routine well-child visits. These should take place at 1, 3, 5-6, 8-9, 12, 18, 24, 36 and 60-72 months of age. However, parents do not always comply with this schedule and visits may take place at different ages. For the purpose of the present report, only visits taking place at $\pm 15 \mathrm{~d}$ of the scheduled visit in the first year of life, \pm 1 month in the second year, \pm 2 months at 24 months, \pm 3 months at 36 months and \pm 6 months at 5-6 years of age, were considered. As testified by the 5-6, 8-9 and 60-72 months intervals, the schedules for the wellchild visits were slightly different in the three regions; in addition, in Tuscany there was no scheduled visit at 24 months. For the pilot test of the surveillance system, each paediatrician gathered data for six months, between November 2008 and August 2009, using his or her own clinical software, specifically adapted for the purpose of the study. The collected data were sent to the research coordinator either directly, using customised EpiData files, or indirectly through the software providers.

The indicators included in the surveillance system were split into four categories: anthropometric indicators (measured weight and height of the children and referred weight and height of their parents), diet (breast-feeding and complementary feeding, including the consumption of milk products, cereals, meat, fish, pulses, eggs, fruit, vegetables and sugar-sweetened beverages (SSB)), physical activity (number of days in a week in which moderate-to-vigorous physical activity was carried out for more than $1 \mathrm{~h}$, outside school hours for children and outside working hours for parents) and sedentarity (number of days in a week in which children spent more than $2 \mathrm{~h}$ watching television or playing video games); and sociodemographic variables for parents (age, education, area of residence, whether or not their child was in child care or in nursery school). Standard instructions were issued to paediatricians for measuring weight and height. Breast-feeding and complementary feeding were investigated using the indicators, definitions and methods recommended by $\mathrm{WHO}^{(9,10)}$. The consumption of breast milk and of different food groups was investigated using a $24 \mathrm{~h}$ recall. Data gathered with a different recall period were excluded from the final analysis. Daily frequencies were added for fruit, vegetables and SSB. Moderateto-vigorous physical activity was defined as one that would lead to an increase in breathing and heart rate, and to a feeling of increased warmth, accompanied by sweating on hot or humid days.

The tool used for data collection varied with the age of the child. For example, questions on breast-feeding were included only for children up to 3 years of age, while physical activity and sedentary behaviours were investigated from 2 years of age onwards. Sociodemographic data were gathered only at the first contact. The denominators used to estimate rates varied for different variables and age groups. All of the tools were pilot tested before use and were illustrated to participating paediatricians during a one-day training course. At the end of the data collection period, paediatricians were asked to fill in an online questionnaire to express their opinions on the surveillance tool, the reliability of questions and answers, the time spent to gather data and the willingness to continue on a routine basis.

For statistical analysis, all records with available data were used, except for those with missing dates. Anthropometric indicators were calculated using the WHO growth standards and WHO's Anthro and Anthro plus software; results are reported as $Z$-scores ${ }^{(11)}$. The associations between categorical or dichotomous variables were analysed using the $\chi^{2}$ test and the $\chi^{2}$ test for trend. For many variables we used internationally accepted categories (e.g. feeding, anthropometry, parental education). For others, we relied on recommendations issued by wellknown professional bodies (e.g. physical activity, sedentary 
behaviours) or simply categorised as yes or no (e.g. consumption of vegetables or SSB). Differences in means between groups were analysed using the $t$ test or ANOVA if data were normally distributed, the Mann-Witney or Kruskall-Wallis test if the normality assumption was not satisfied. The Bonferroni correction was used to account for multiple testing. Trends in means between ordered groups were assessed using the non-parametric test for trend across ordered groups developed by Cuzick. To adjust for the possible effect of clustering, two-level (region and paediatrician) mixed-effect logistic regression modelling was used to identify the independent effect of sociodemographic variables on the indicators.

\section{Results}

The analysis refers to 32915 well-child visits of 26898 children (of whom $52 \%$ were male), after the exclusion of 3592 (about 10\%) visits that were out of the age range for that visit or with very incomplete data. During the six months of data collection, the majority of children underwent one $(71.4 \%)$ or two $(21.9 \%)$ visits, the remaining $6 \cdot 7 \%$ were visited three times; multiple visits took place only in infants. Lower denominators were used for the analysis of feeding practices, due to the exclusion of data collected with a recall period different from $24 \mathrm{~h}$ in one of the regions. Table 1 shows the number of visits by age and region. As far as the characteristics of parents are concerned, the mean maternal and paternal ages were very similar, about 34.5 and 37.5 years, respectively, in Friuli Venezia Giulia and Tuscany, but were lower, 33 and 36 years, in Puglia $(P<0 \cdot 001)$. Also the proportion of mothers and fathers with high school or higher education was similar, about $77 \%$ and $65 \%$, respectively, in Friuli Venezia Giulia and Tuscany, and 56\% and 49\% in Puglia $(P<0.001$ between this and the other two regions). There was a significant difference $(P<0 \cdot 001)$ between Puglia and the other two regions in the reported prevalence of parental overweight $\left(\mathrm{BMI}=25 \cdot 0-29 \cdot 9 \mathrm{~kg} / \mathrm{m}^{2}\right)$ and obesity (BMI $\geq 30 \cdot 0 \mathrm{~kg} / \mathrm{m}^{2}$ ). In Puglia, $23 \%$ of mothers and $46 \%$ of fathers were overweight, $8 \%$ and $11 \%$ were obese; in Tuscany, $16 \%$ and $41 \%$ were overweight, $5 \%$ and $8 \%$ were obese; in Friuli Venezia Giulia, 14\% of mothers and $42 \%$ of fathers were overweight, $5 \%$ and $9 \%$ were obese. Only $3 \%$ of infants were in child care at 9 months and $9 \%$ at 12 months; at 36 months of age $64 \%$ of children were in child care or in nursery school, with no significant differences among regions.

\section{Growth}

Figure 1 shows the mean BMI Z-scores and standard deviations of the study children at different ages. Compared with the WHO standards, BMI was lower at 1 month (mean $Z$-score $-0 \cdot 32$, SD $1 \cdot 03$ ) and 3 months (mean $Z$-score $-0 \cdot 21$, SD $1 \cdot 05)$, and higher from 8-9 months onwards, reaching a
Table 1 Visits available for analysis, by age and region, from offices of 179 paediatricians in three Italian regions, November 2008-August 2009

\begin{tabular}{lcrrr}
\hline Age (months) & Friuli Venezia Giulia & Tuscany & Puglia & Total \\
\hline 1 & 741 & 567 & 1636 & 2944 \\
3 & 872 & 831 & 2047 & 3750 \\
$5-6$ & 896 & 1065 & 2596 & 4557 \\
$8-9$ & 879 & 1070 & 1821 & 3770 \\
12 & 853 & 919 & 2975 & 4747 \\
18 & 690 & 872 & 2166 & 3728 \\
24 & 615 & 0 & 2106 & 2721 \\
36 & 564 & 695 & 1830 & 3089 \\
$60-72$ & 320 & 814 & 2475 & 3609 \\
Total & 6430 & 6833 & 19652 & 32915 \\
\hline
\end{tabular}

mean Z-score of $0 \cdot 62(\mathrm{sD} 1 \cdot 37)$ at $60-72$ months of age. All of the differences between the BMI of the study children and the WHO standards were statistically significant $(P<0.05)$, except at 5-6 months of age. There were no significant differences by sex; at age 60-72 months boys had a slightly higher BMI $Z$-score than girls, but the difference was not statistically significant.

\section{Diet}

Figure 2 shows the feeding patterns up to 36 months of age. At $8-9$ months, more than $50 \%$ of infants continued breastfeeding; more than $30 \%$ were still breast-fed at 12 months, with 3\% continuing up to 36 months. At 5-6 months, almost $20 \%$ of infants were exclusively breast-fed. At 1 month of age, $8 \%$ of infants were given non-nutritive liquids in addition to breast milk, half of them being SSB such as chamomile tea. At 3 months, the percentage of infants given non-nutritive liquids increased to $11 \%$, with $5 \%$ for SSB. The intake of SSB reached a peak of $51 \%$ at $60-72$ months ( $\chi^{2}$ for trend, $P<0 \cdot 001$ ). A small percentage of infants (3\%) were already receiving complementary foods at 3 months. At 5 and 6 months, $57 \%$ and $85 \%$ of infants, respectively, were being given complementary foods, mostly milk products, cereals and meat. At 8-9 months, all infants were on complementary foods. The prevalence of milk products, cereals and meat in complementary feeding patterns did not change with age, the consumption of other categories of food remaining low (Fig. 3). A low percentage of children had been given fresh milk, cows' milk or other, by or before 12 months of age: $6 \%$ at $8-9$ and $36 \%$ at 12 months, when the consumption of cows' milk was significantly higher in Friuli Venezia Giulia than in Tuscany ( $47 \%$ v. 25\%, $P<0 \cdot 0001)$. The intake of fruit and vegetables decreased between 8-9 (93\%) and 60-72 (83\%) months ( $\chi^{2}$ for trend, $P<0.001$ for both fruit and vegetables). Percentages were much lower if the recommended intake of five portions daily is considered: only $8 \%$ and $10 \%$ of children at 36 and 60-72 months, respectively, were taking the recommended amount of fruit and vegetables.

Multilevel logistic regression analysis was used to look at the association between consumption of fruit, vegetables and SSB, and other variables. Table 2 shows that the higher 


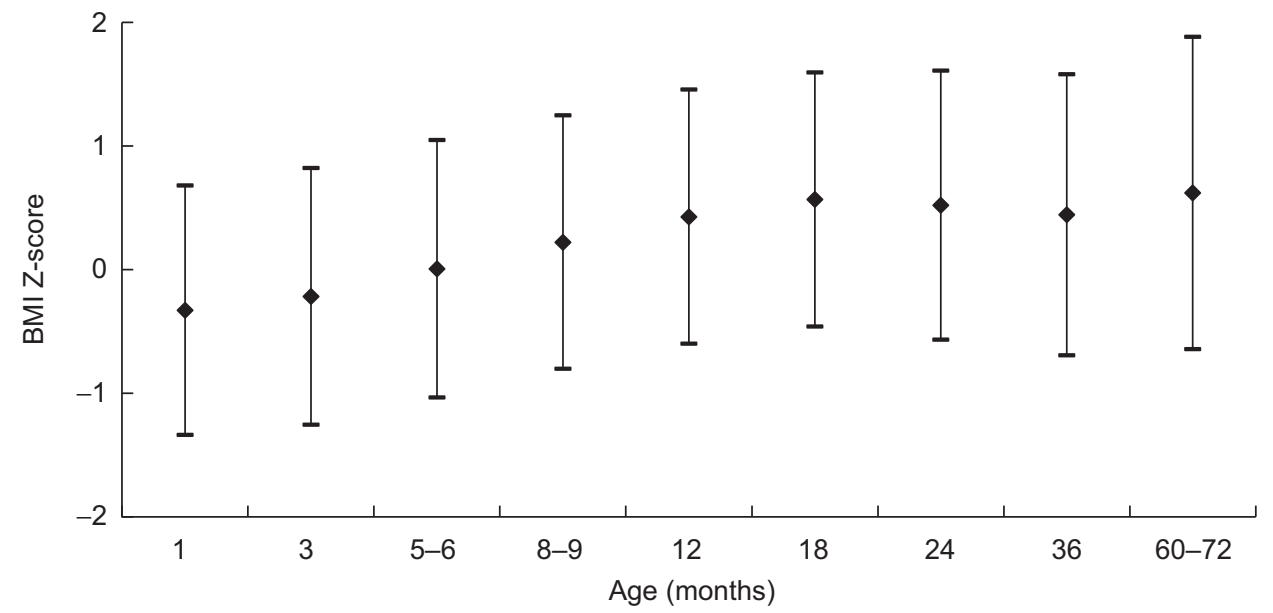

Fig. 1 BMI Z-score, by age, compared with the WHO standards (mean Z-score = 0, sD 1), among pre-school children ( $n 26898$; 32915 well-child visits) from three regions of Italy (Friuli Venezia Giulia, Tuscany and Puglia), November 2008-August 2009. Values are means with standard deviations represented by vertical bars

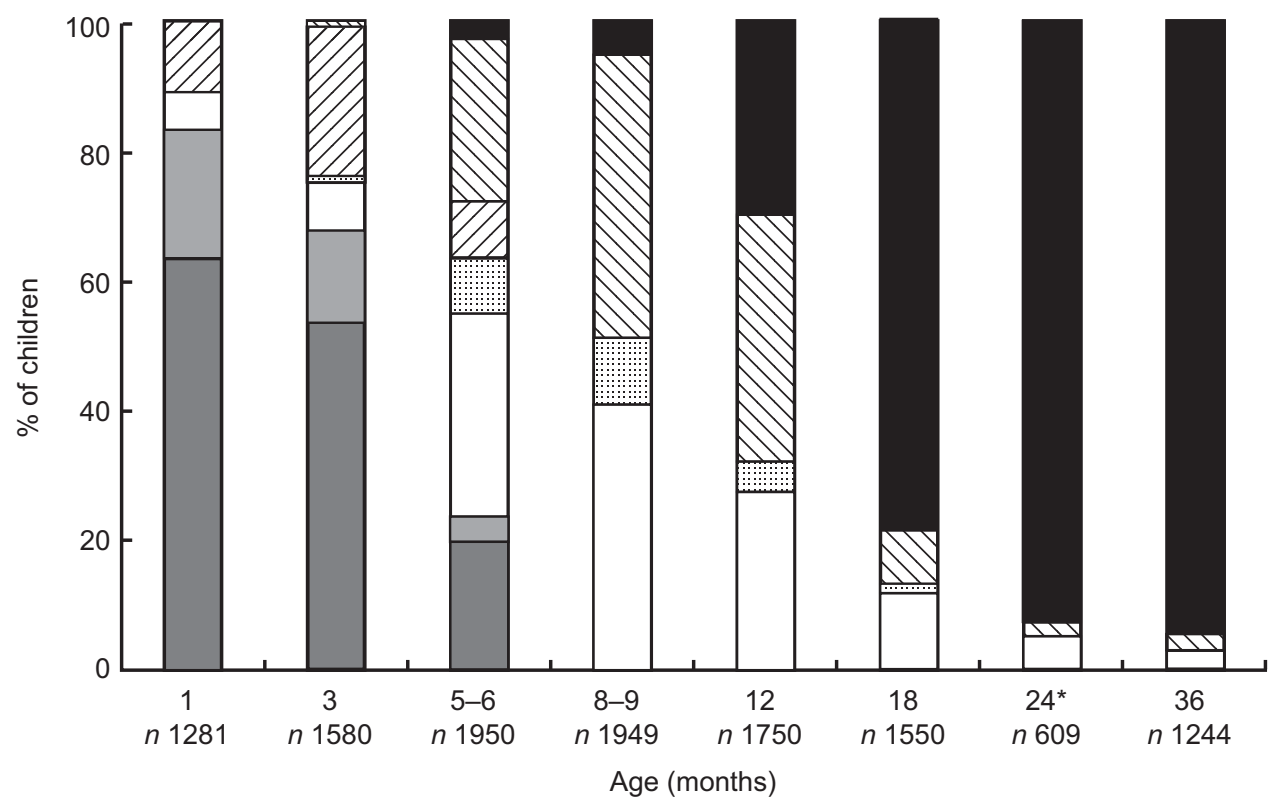

Fig. 2 Feeding practices ( $\square$, EBF; $\square, B F+F F ; \square, B F+C F ; \square, B F+F F+C F ; \square, F F ; \mathbb{N}, F F+C F ; \square$, CF) in the first 36 months of life among pre-school children ( $n 26898 ; 32915$ well-child visits) from three regions of Italy (Friuli Venezia Giulia, Tuscany and Puglia), November 2008-August 2009. *Data refer only to Friuli Venezia Giulia (EBF, exclusive breast-feeding; BF, breast-feeding; FF, formula feeding; CF, complementary feeding; note that CF at 1 and 3 months stands for non-nutritive fluids)

the level of maternal education, the higher the odds, from 8-9 to 18 months, of eating at least one portion of vegetables daily, and the lower the odds of drinking SSB at almost all ages, independently of maternal age and parental BMI. The consumption of vegetables was significantly associated with higher maternal age only at 8-9 and 24 months (both adjusted OR (aOR) =1.06; $P=0.016$ and $P=0 \cdot 011$, respectively). The consumption of fruit was significantly associated with higher maternal education only at 12 months $(\mathrm{aOR}=2 \cdot 72 ; P=0 \cdot 005)$ and 18 months $(\mathrm{aOR}=1 \cdot 92 ; P=0 \cdot 048)$. BMI $>25 \cdot 0 \mathrm{~kg} / \mathrm{m}^{2}$ in both parents was associated with lower odds of eating vegetables at $8-9$ months $(\mathrm{aOR}=0.54 ; P=0.013)$ and higher odds of drinking SSB at 36 months (aOR $=1 \cdot 53 ; P=0 \cdot 022)$. Maternal age was significantly associated with SSB consumption at almost all ages from 8-9 months onwards (aOR ranging from 0.96 to $0.93, P$ ranging from $0 \cdot 008$ to $<0 \cdot 001$ ). There were no other significant associations.

\section{Pbysical activity}

The percentage of 36-month-old children whose parents reported $0-1,2-4$ and 5-7 d/week with more than $1 \mathrm{~h}$ of 


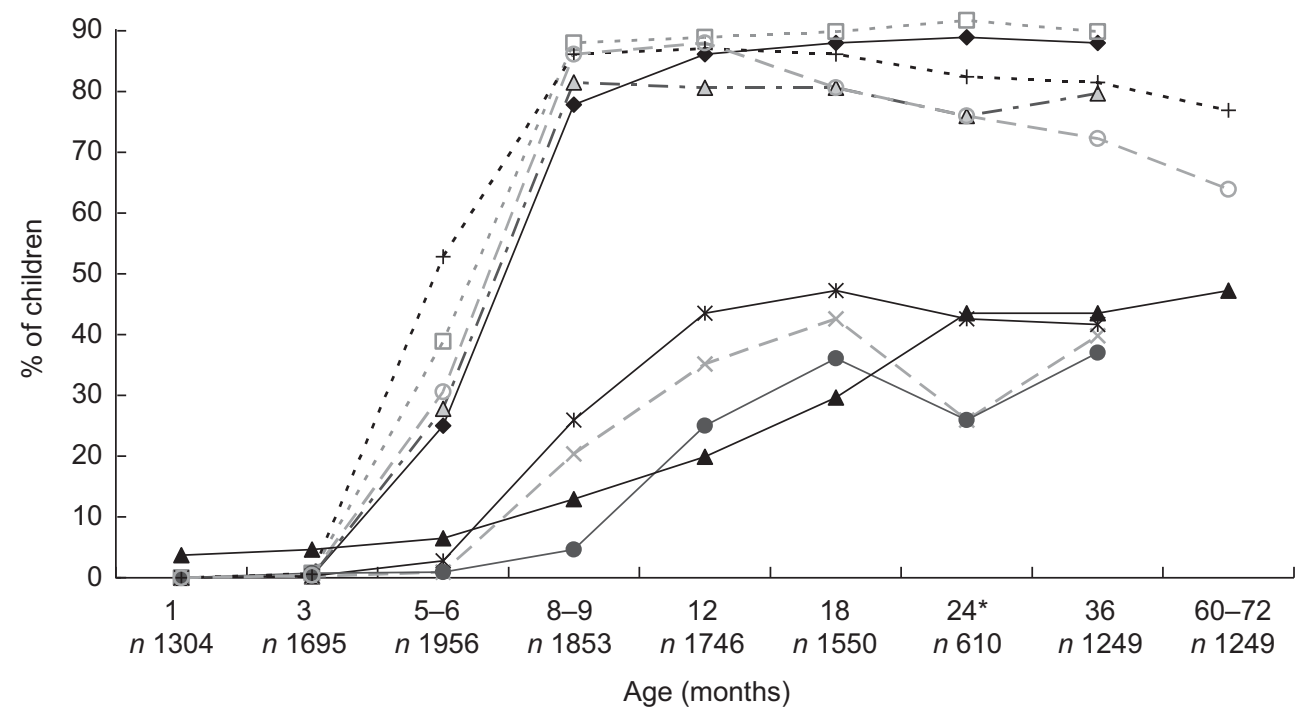

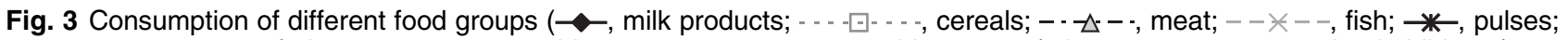
-0 , eggs; - - - - - - , fruit; - - $\ominus--$, vegetables; $-<$, sugar-sweetened beverages), by age, among pre-school children ( $n 26$ 898; 32915 well-child visits) from three regions of Italy (Friuli Venezia Giulia, Tuscany and Puglia), November 2008-August 2009. *Data refer only to Friuli Venezia Giulia

Table 2 Association between maternal education and consumption of vegetables and sugar-sweetened beverages (SSB), in different age groups, among pre-school children ( $n 26$ 898; 32915 well-child visits) from three regions of Italy (Friuli Venezia Giulia, Tuscany and Puglia), November 2008-August 2009. Multilevel logistic regression; odds ratios adjusted for maternal age and parental BMI with 'up to middle school' as reference category.

\begin{tabular}{|c|c|c|c|c|c|}
\hline \multirow[b]{2}{*}{ Age (months) } & \multirow[b]{2}{*}{$n$} & \multicolumn{2}{|c|}{ Consumption of vegetables* (yes $v$. no) } & \multicolumn{2}{|c|}{ Consumption of SSBt (yes v. no) } \\
\hline & & High school diploma & University degree & High school diploma & University degree \\
\hline $8-9$ & 1767 & $1 \cdot 39$ & $2 \cdot 77 \|$ & $0 \cdot 52 \|$ & $0 \cdot 33 \|$ \\
\hline 12 & 1560 & $1.78 \S$ & $1.95 \S$ & $0.65 \S$ & $0.56 \$$ \\
\hline 18 & 1365 & $1 \cdot 20$ & $2 \cdot 37 \|$ & $0.71 \S$ & $0.54 \S$ \\
\hline $24 \ddagger$ & 462 & $1.90 \S$ & $1.99 \S$ & $0.61 \S$ & $0.54 \S$ \\
\hline 36 & 1060 & $1 \cdot 17^{\circ}$ & $1 \cdot 27$ & $0.57 \|$ & $0 \cdot 46 \|$ \\
\hline 60-72 & 1009 & $1 \cdot 10$ & 1.50 & $0.60 \S$ & $0.46 \|$ \\
\hline
\end{tabular}

${ }^{*}$ Consumption of at least one portion daily.

tConsumption at least once daily.

‡Data refer only to Friuli Venezia Giulia and Puglia.

$\S$ Wald $P<0.05$ compared with reference category.

$\|$ Wald $P<0.001$ compared with reference category.

moderate-to-vigorous physical activity outside school hours was $83 \%, 7 \%$ and $9 \%$, respectively. At 60-72 months of age the percentage was $57 \%, 35 \%$ and $8 \%$. In line with children's data for physical activity, a high percentage of parents led a sedentary life: $70 \%$ of mothers and $58 \%$ of fathers reported less than $1 \mathrm{~d} /$ week with more than $1 \mathrm{~h}$ of moderate-to-vigorous physical activity. Children started spending time watching television from very early on in life. Fig. 4 shows that at 12 months $89 \%$ of children had never watched television or played video games for more than $2 \mathrm{~h} / \mathrm{d}$ during the week before the visit. This percentage decreased with age reaching $27 \%$ at $60-72$ months $\left(\chi^{2}\right.$ for trend, $P<0 \cdot 001$ ), with $32 \%$ of children watching television or playing video games for more than $2 \mathrm{~h} / \mathrm{d}$, every day. The multilevel analysis of Table 3 shows that the higher the education of the mothers, the lower the chance that their children watch television or play video games for more than $2 \mathrm{~h} / \mathrm{d}$ more than twice weekly; this association was statistically significant $(P<0.05$ or $P<0.001)$ at all ages except at 12 and 24 months. Watching television or playing video games for more than $2 \mathrm{~h} / \mathrm{d}$ more than twice weekly was also associated with parental BMI $>25 \cdot 0 \mathrm{~kg} / \mathrm{m}^{2}$ from 24 months of age onwards at multilevel analysis.

\section{Comments by paediatricians}

A total of 103 paediatricians (58\%) filled in the online questionnaire, twenty-five in Friuli Venezia Giulia, twentynine in Tuscany and forty-nine in Puglia. According to $62 \%$ of the 103 paediatricians, the surveillance system covered between $75 \%$ and $100 \%$ of the children enrolled in their practices; the remaining 38\% estimated a lower coverage. The collection of data lasted about 8 min during the first visit and about $5 \mathrm{~min}$ at subsequent visits. The majority of 


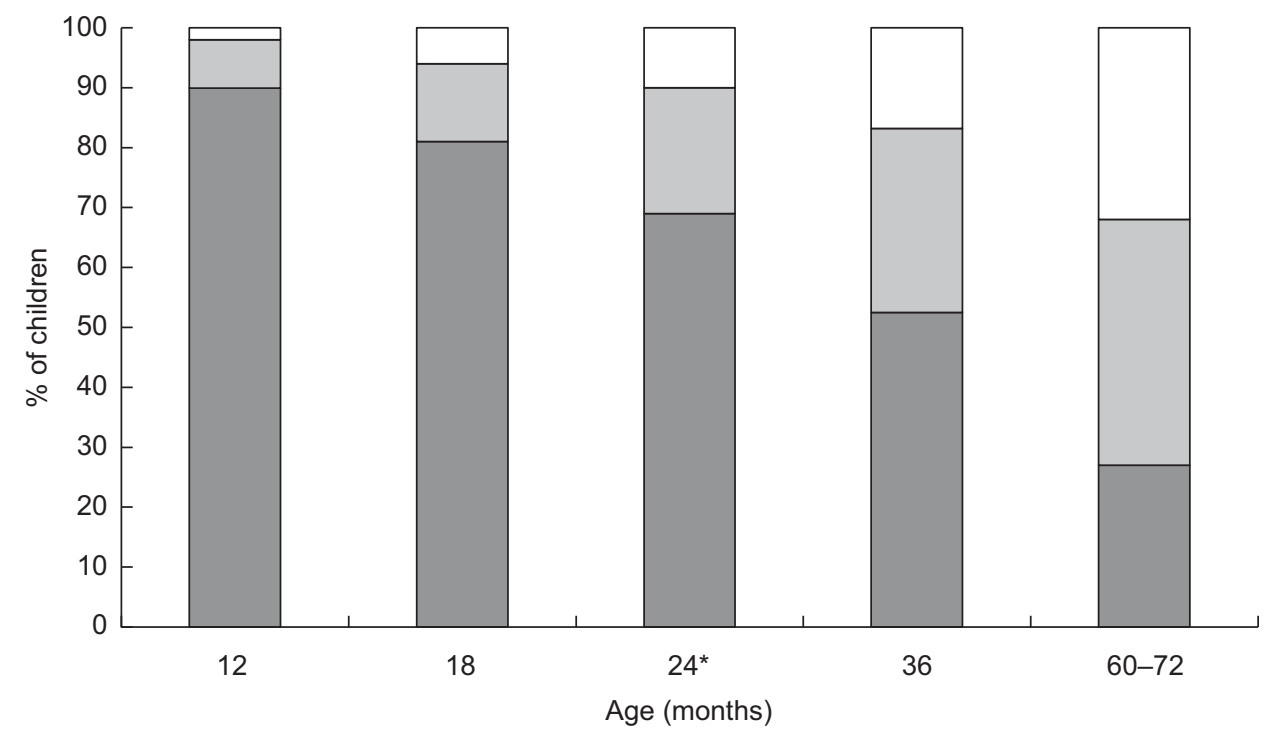

Fig. 4 Number of days per week $(\square, 0 ; \square, 1-6 ; \square, 7)$ in which children spent $2 \mathrm{~h} / \mathrm{d}$ or more watching television or playing video games, by age, among pre-school children ( $n 26$ 898; 32915 well-child visits) from three regions of Italy (Friuli Venezia Giulia, Tuscany and Puglia), November 2008-August 2009. *Data refer only to Friuli Venezia Giulia

Table 3 Association between maternal education and parental BMI and spending $2 \mathrm{~h} / \mathrm{d}$ or more watching television or playing video games, in different age groups, among pre-school children ( $n 26$ 898; 32915 well-child visits) from three regions of Italy (Friuli Venezia Giulia, Tuscany and Puglia), November 2008-August 2009. Multilevel logistic regression; odds ratios adjusted for maternal age with 'up to middle school' and 'both parents with $\mathrm{BMI} \leq 25 \cdot 0 \mathrm{~kg} / \mathrm{m}^{2}$ ' as reference categories

\begin{tabular}{|c|c|c|c|c|c|}
\hline \multirow[b]{2}{*}{ Age (months) } & \multirow[b]{2}{*}{$n$} & \multicolumn{4}{|c|}{ Watching television or playing video games for $2 \mathrm{~h} / \mathrm{d}$ or more ( $>2 \mathrm{~d} /$ week $v . \leq 2 \mathrm{~d} /$ week) } \\
\hline & & $\begin{array}{l}\text { High school } \\
\text { diploma }\end{array}$ & $\begin{array}{l}\text { University } \\
\text { degree }\end{array}$ & $\begin{array}{l}\text { One parent with } \\
\mathrm{BMI}>25.0 \mathrm{~kg} / \mathrm{m}^{2}\end{array}$ & $\begin{array}{l}\text { Both parents with } \\
\text { BMI }>25.0 \mathrm{~kg} / \mathrm{m}^{2}\end{array}$ \\
\hline 12 & 4484 & 0.83 & 0.63 & $1 \cdot 00$ & $1 \cdot 02$ \\
\hline 18 & 3492 & $0.60 t$ & $0.59 \ddagger$ & 1.01 & $1 \cdot 37$ \\
\hline $24^{*}$ & 2536 & 0.94 & 0.73 & $1 \cdot 11$ & $1 \cdot 42 \ddagger$ \\
\hline 36 & 2870 & $0.65 t$ & $0.66 \neq$ & 1.07 & $1 \cdot 36 \neq$ \\
\hline $60-72$ & 3456 & $0 \cdot 87$ & $0.66+$ & $1 \cdot 31 \ddagger$ & $1 \cdot 29 \ddagger$ \\
\hline
\end{tabular}

*Data refer only to Friuli Venezia Giulia and Puglia.

+Wald $P<0.001$ compared with reference category.

$\ddagger$ Wald $P<0.05$ compared with reference category.

paediatricians rated the data gathered as reliable, except for referred parental height and weight. As far as diet and physical activity are concerned, about $90 \%$ of the respondents considered the answers reliable, but the percentage went down to about $70 \%$ when sedentarity was investigated. The reliability of data was considered worse when parents were foreigners and could not speak Italian. About $90 \%$ of the paediatricians regarded the surveillance system as feasible on a routine basis.

\section{Discussion}

To our knowledge, the present paper is the first to report results of a surveillance system on growth, diet and physical activity in pre-school children based on routine data collected at well-child visits. Our report shows that such a system is feasible and does not represent a burden for family paediatricians or other health professionals in charge of well-child visits. Whether such a surveillance system is sustainable is yet to be established and will need further research. Its usefulness for planning and evaluation of activities, including those aimed at preventing overweight and obesity, will depend on national and local health authorities. These will be more likely to use the results provided by such a surveillance system if they consider them valid and reliable in terms of completeness and accuracy. Completeness will depend on the number of paediatricians involved and their coverage for well-child visits. Accuracy will vary by the factor being explored (see below regarding how difficult it is to reliably measure physical activity in pre-school children) and will be directly linked to the use of standard methods of data collection; regular comparisons with gold standards will be needed. Despite the limitations of our study, including the fact that some data had to be excluded from the analysis, that 
paediatricians participated on a voluntary basis and that well-child visits did not cover $100 \%$ of the child populations, we consider our results representative; the analysis of the sociodemographic variables shows that their distribution in our sample is similar to the one in the general population of Italy, with the typical north-to-south gradient. Parents living in the north are older and have a higher level of education than parents living in the south $^{(12)}$. Also, in our sample less than $20 \%$ of young children up to 2 years of age were in child care, with the same north-to-south gradient found by other surveys ${ }^{(12)}$. Finally, our results show that children and parents living in the south have higher rates of overweight and obesity compared with those living in the north, a difference which is also reported by other sources ${ }^{(3,4,13)}$.

Compared with the WHO growth standards, the infants in our sample had lower BMI at 1 and 3 months of age, but higher BMI from 8-9 months onwards. This may be due to the fact that the rates of exclusive breast-feeding up to 3 months were lower than those of the infants used to construct the WHO curves, who were all exclusively breast-fed for at least 4 months by definition $^{(11)}$, and to the likely excess of energy and protein associated with the intake of formula and other milks. The rates of breast-feeding in our sample, however, were generally higher than those previously reported for Italy $^{(14)}$. Yet they were lower than those recently reported for Trieste, the main town in Friuli Venezia Giulia ${ }^{(15)}$. Our results show that rates of exclusive breast-feeding are generally low during the first 6 months of life, contrary to global and national recommendations ${ }^{(16,17)}$. To our knowledge, our report is the first in Italy, and probably one of the few worldwide, on rates of breast-feeding up to 36 months. The recent introduction on the market of the so-called 'growing up formula' probably explains the continuation of formula feeding after the first year of life, with a minority of children (3\%) continuing up to 3 years, even if there is no evidence of any beneficial effect of these products. The study results show that most infants are introduced to complementary foods at around 6 months of age. Two main food groups characterize the diet of infants and children: one group is introduced around 5-6 months of age and includes cereals, fruit, vegetables, meat and milk products; the other is introduced later on, around 8-9 months, and includes eggs, fish and pulses. This pattern is probably the result of a deeply rooted fear of allergic reactions that is not based on evidence ${ }^{(18)}$. Alternatively, parents may have decided to start with mildly tasting foods that infants like, leaving the stronger tasting foods for later. After introduction, the consumption of the various food groups seems to remain constant. This is not true for the consumption of fruit and vegetables however, which tends to decrease gradually and fails to meet the 'five a day' recommendation, with only $8 \%$ of children achieving that standard at 36 and 60-72 months of age; this is in line with data (9\%) from surveys in children 8-9 years old ${ }^{(4)}$. We report a high consumption of SSB, starting at low levels in early infancy and increasing worryingly over the following years. At 6 years of age, $44 \%$ of children consume SSB at least once daily, in line with national data at 8-9 years of age $(48 \%)^{(4)}$. Our data show that the low consumption of fruit and vegetables and the high consumption of SSB is associated with family characteristics, such as maternal age and education, and parental BMI, as reported by a recent review of the literature ${ }^{(6)}$.

Measuring moderate-to-vigorous physical activity in pre-school children is far from easy, even in a research setting ${ }^{(19)}$. The questionnaires we used may have overestimated the actual frequency and intensity of physical activity in children ${ }^{(20)}$. There may also have been biases in the interpretation of the questions by paediatricians and parents. Direct observation would yield more reliable results ${ }^{(21)}$, but this is not feasible in a well-child clinic setting. The limited evidence of reliability of parental reporting represented a great limitation for our study and will be a big challenge for any future surveillance system of physical activity in this age group ${ }^{(22)}$. Even considering these limitations, our results indicate a low engagement of our sample of children in moderate-to-vigorous physical activity, certainly less than the at least $60 \mathrm{~min} / \mathrm{d}$ currently recommended for children $4-5$ years old ${ }^{(23,24)}$. This low engagement of our children is indirectly confirmed by the low engagement reported by their parents. There is some evidence of a correlation between the levels of physical activities of young children and the attitude to physical activity of their parents ${ }^{(19,25)}$. With regard to sedentarity, there are guidelines recommending parents to limit their children's total daily screen time to no more than 1 to $2 \mathrm{~h}$ of quality programming for children 2 years of age and older ${ }^{(26)}$. Despite the lack of evidence of effectiveness for interventions aiming at reducing screen time ${ }^{(27)}$, the recommendation is appropriate given the growing evidence of an association between childhood obesity and television viewing ${ }^{(6)}$. Our study yields alarming results: six out of ten children over 2 years of age and $20 \%$ of infants and young children under 2 years of age spend $2 \mathrm{~h} / \mathrm{d}$ or more watching television or playing video games at least once weekly. In addition, $25 \%$ of children between 2 and 6 years of age are reported to watch television or play video games for $2 \mathrm{~h} / \mathrm{d}$ or more, every day. These behaviours are associated with low maternal education at all ages, while high parental BMI seems associated with sedentary habits only at 3 and 6 years of age.

\section{Conclusion}

Our results provide a preliminary, though partial, picture of growth, diet and physical activity among pre-school children in Italy. They show that there is much to be done to meet the recommendations for the development of 
healthy lifestyles from early childhood. The setting up of a sustainable surveillance system based on our pilot test may help gain valuable information for the planning and evaluation of public health policies and interventions aimed at reducing the burden of disease in future generations. In doing so, an equity lens should be used, given the associations we found with equity stratifiers such as maternal education and area of residence.

\section{Acknowledgements}

Sources of funding: The project was fully financed by the Centre for the Control of Diseases of the Italian Ministry of Health in the framework of the programme 'Gaining Health'. Conflict of interest: All the authors declare that they have no conflicts of interest. Authors' contributions: A.C., C.C., A.M., M.C. and M.G. contributed to the project design. C.C. developed the training tools, with contributions from A.M., M.C. and M.G. C.C., A.M., M.C., M.G. and A.C. organized and ran the training courses. A.C. coordinated the whole project; A.M., M.C. and M.G. were the coordinators in Friuli Venezia Giulia, Puglia and Tuscany. M.M., P.P., C.C., A.M. and A.C. carried out the statistical analysis of the data and helped interpreting the results. C.C., A.M. and P.P. wrote the drafts of the paper that were revised by all authors. A.C. wrote the final version of the paper. All the other members of the project group supported the activities of the national and regional coordinators. All authors read and approved the final manuscript. Acknowledgements: The authors would like to express their gratitude to Silvia Colitti, Paolo D’Argenio, Roberto D'Elia, Daniela Galeone, Donato Greco, Letizia Zanetti (Centre for the Control of Diseases, Italian Ministry of Health) and to Emanuela Balocchini (technical coordination between the Italian Ministry of Health and the Italian Regions) for their support. Finally, and most importantly, the authors wish to thank the 179 family paediatricians who participated voluntarily to the collection of data; without their commitment the project would have not come to a successful conclusion. Ethical approval: Ethical approval was not required as all data were gathered by family paediatricians during their routine daily activities.

Members of the Project Group:

- Alessandro Bavcar, Claudia Carletti, Adriano Cattaneo, Paola D'Acapito, Sara Daris, Alessandra Knowles, Anna Macaluso, Mariarosa Milinco, Marcella Montico, Paola Pani (Health Services Research and International Health, Institute for Maternal and Child Health IRCCS Burlo Garofolo, Trieste, Italy);

- Paolo Lubrano, Daniela Rosenwirth, Daniele Venier (Friuli Venezia Giulia Region);

- Stefano Castelli, Francesco De Feo, Mariano Giacchi, Valdo Flori, Andrea Pamolli, Valentina Pilato, Rita Simi (Tuscany Region);
- Margherita Caroli, Teresa Cazzato, Maria Rosaria Filograna, Ruggiero Piazzolla (Puglia Region).

\section{References}

1. Branca F, Nikogosian H \& Lobstein T (editors) (2007) The Challenge of Obesity in the WHO European Region and the Strategies for Response. Copenhagen: WHO Regional Office for Europe.

2. Lobstein T, Baur L \& Uauy R (2004) Obesity in children and young people: a crisis in public health. Obes Rev $\mathbf{5}$, Suppl. 1, 4-104.

3. Istituto Superiore di Sanità (2010) Rapporto Nazionale Passi 2010. Rome: ISS; available at http://www.epicentro.iss.it/ passi/R2010Indice.asp

4. Istituto Superiore di Sanità (2011) Okkio alla Salute 2010: sintesi dei risultati. Rome: ISS; available at http://www. epicentro.iss.it/okkioallasalute/pdf/sintesi\%20OKkio\%20alla \%20SALUTE\%202010_def.pdf.

5. Cattaneo A, Monasta L, Stamatakis E et al. (2010) Overweight and obesity in infants and pre-school children in the European Union: a review of existing data. Obes Rev 11, 389-398.

6. Monasta L, Batty GD, Cattaneo A et al. (2010) Early-life determinants of overweight and obesity: a review of systematic reviews. Obes Rev 11, 695-708.

7. Cross-Government Obesity Unit, Department of Health \& Department of Children Schools and Families (2008) Healthy Weight, Healthy Lives: A Cross-Government Strategy for England. London: HM Government.

8. Centro per il Controllo delle Malattie (2006) Guadagnare Salute: Rendere Facili le Scelte Salutari. Rome: Ministero della Salute.

9. World Health Organization (2009) Indicators for Assessing Infant and Young Child Feeding Practices: Part 1 Definitions. Geneva: WHO.

10. World Health Organization (2010) Indicators for Assessing Infant and Young Child Feeding Practices: Part 2 Measurement. Geneva: WHO.

11. WHO Multicentre Growth Reference Study Group (2006) WHO Child Growth Standards: Length/Height-for-Age, Weight-for-Age, Weight-for-Length, Weight-for-Height and Body Mass Index-for-Age: Methods and Development. Geneva: WHO.

12. Istituto Nazionale di Statistica (2005) La Situazione del Paese nel 2004. Rome: ISTAT.

13. Binkin N, Fontana G, Lamberti A et al. (2010) A national survey of the prevalence of childhood overweight and obesity in Italy. Obes Rev 11, 2-10.

14. Giovannini M, Riva E, Banderali G et al. (2004) Feeding practices of infants through the first year of life in Italy. Acta Paediatr 93, 492-497.

15. Carletti C, Pani P, Knowles A et al. (2011) Breastfeeding to 24 months of age in the northeast of Italy: a cohort study. Breastfeed Med 6, 177-182.

16. World Health Organization (2002) Global Strategy for Infant and Young Child Feeding. Geneva: WHO.

17. Ministero della Salute (2008) Linee di indirizzo nazionali sulla protezione, la promozione ed il sostegno dell'allattamento al seno. Gazzetta Ufficiale N. 32 del 7 Febbraio 2008, Roma.

18. Agostoni C, Decsi T, Fewtrell M et al. (2008) Complementary feeding: a commentary by the ESPGHAN Committee on Nutrition. J Pediatr Gastroenterol Nutr 46, 99-110.

19. Dowda M, Pfeiffer KA, Brown WH et al. (2011) Parental and environmental correlates of physical activity of children attending preschool. Arch Pediatr Adolesc Med 165, 939-944.

20. Basterfield L, Adamson AJ, Parkinson KN et al. (2008) Surveillance of physical activity in the UK is flawed: validation 
of the Health Survey for England Physical Activity Questionnaire. Arch Dis Child 93, 1054-1058.

21. McIver KL, Brown WH, Pfeiffer KA et al. (2009) Assessing children's physical activity in their homes: the observational system for recording physical activity in children-home. J Appl Behav Anal 42, 1-16.

22. Purslow LR, van Jaarsveld CH, Semmler C et al. (2009) Validity and prognostic value of parental ratings of children's activity. Prev Med 49, 28-31.

23. National Association for Sport and Physical Education (2009) Active Start: A Statement of Physical Activity Guidelines for Children Birth to Five Years, 2nd ed. Reston, VA: NASPE Publications.
24. Department of Health and Ageing (2010) Get Up \& Grow: Healthy Eating and Physical Activity for Early Childhood. Canberra: Australian Government.

25. Loprinzi PD \& Trost SG (2010) Parental influences on physical activity behavior in preschool children. Prev Med 50, 129-133.

26. Council on Communications and Media, Brown A (2011) Media use by children younger than 2 years. Pediatrics 128, 1040-1045.

27. Wahi G, Parkin PC, Beyene J et al. (2011) Effectiveness of interventions aimed at reducing screen time in children: a systematic review and meta-analysis of randomized controlled trials. Arch Pediatr Adolesc Med 165, 979-986. 